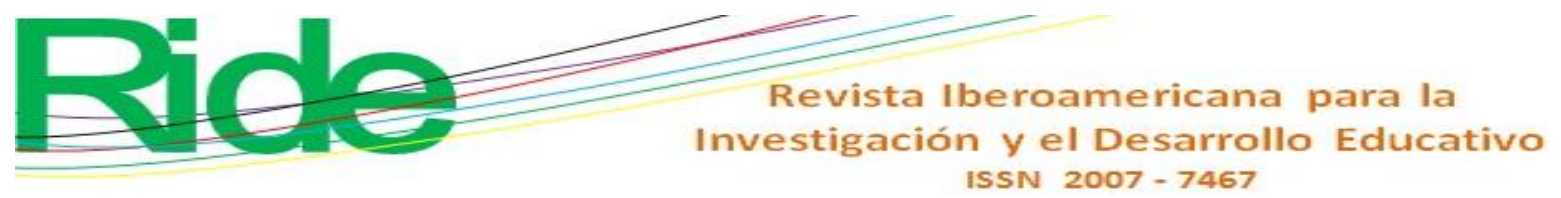

https://doi.org/10.23913/ride.v10i19.515

Artículos Científicos

\title{
Competencias para el uso de las TIC en estudiantes de educación superior: un estudio de caso
}

ICT Competences in students of higuer education, a case study

\begin{abstract}
Competências para o uso de TIC em estudantes do ensino superior: um estudo de
\end{abstract}
caso

Carlos Miguel Amador Ortiz

Instituto Tecnológico José Mario Molina Pasquel y Henríquez, campus Puerto Vallarta, México carlos.amador@vallarta.tecmm.edu.mx https://orcid.org/0000-0001-6654-8448

Leticia Velarde Peña

Instituto Tecnológico José Mario Molina Pasquel y Henríquez, campus Puerto Vallarta, México leticia.velarde@vallarta.tecmm.edu.mx https://orcid.org/0000-0003-1096-1035

\section{Resumen}

En este trabajo se presenta un estudio llevado a cabo en el Instituto Tecnológico José Mario Molina Pasquel y Henríquez (TECMM), campus Puerto Vallarta, en el que se analizan las competencias para el uso de las TIC de estudiantes. Para tal fin, se diseñó un cuestionario tomando en cuenta los dominios e indicadores de los estándares de la International Society for Technology in Education (ISTE, 2007). El estudio se llevó a cabo con una muestra estratificada de 310 estudiantes (de una población de 1605 alumnos). Los resultados más significativos se consiguieron en el uso de las TIC en el TECMM y ciudadanía digital, mientras que los más bajos se obtuvieron en comunicación y colaboración, y creatividad e innovación. Por carrera, los hallazgos más consistentes se encontraron en ingeniería en Sistemas Computacionales, mientras que los más bajos fueron para Gastronomía e ingeniería en Electromecánica. Con estos datos se espera diseñar estrategias que

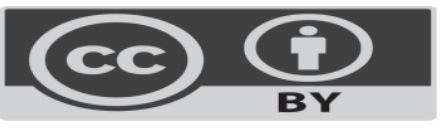




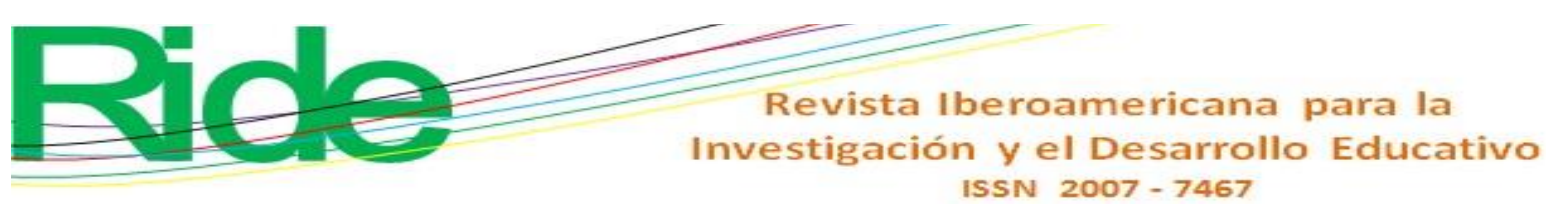

permitan mejorar las capacidades de los alumnos en las áreas con menor desarrollo de competencias.

Palabras clave: competencias tecnológicas, estándares TIC, estrategias de capacitación.

\section{Abstract}

In this paper is presented a study carried out at the Higher Technological Institute of Puerto Vallarta, which analyzes the competences for the use of ICTs for students, for which a questionnaire was designed taking into account the domains and indicators of the standards of the International Society for Technology in Education (ISTE, 2007). The study was carried out with a stratified sample of 310 students from a population of 1605, and the results show a considerable development of competences for the use of ICTs in some domains in relation to others, a relevant aspect to design strategies to improve the skills of students in the less developed areas.

Keywords: technological competences, ICT standards, training strategies.

\section{Resumo}

Este artigo apresenta um estudo realizado no Instituto Tecnológico José Mario Molina Pasquel e Henríquez (TECMM), campus de Puerto Vallarta, no qual são analisadas as competências para o uso das TICs dos alunos. Para tanto, foi elaborado um questionário levando em consideração os domínios e indicadores dos padrões da Sociedade Internacional de Tecnologia em Educação (ISTE, 2007). O estudo foi realizado com uma amostra estratificada de 310 alunos (de uma população de 1605 alunos). Os resultados mais significativos foram alcançados no uso de TIC no TECMM e cidadania digital, enquanto os mais baixos foram obtidos em comunicação e colaboração, criatividade e inovação. Por carreira, os resultados mais consistentes foram encontrados em Engenharia de Sistemas de Computação, enquanto os mais baixos foram para Gastronomia e Engenharia Eletromecânica. Com esses dados, espera-se criar estratégias que permitam aos alunos melhorar suas habilidades nas áreas com menor desenvolvimento de habilidades.

Palavras-chave: habilidades tecnológicas, padrões de TIC, estratégias de treinamento.

Fecha Recepción: Febrero 2019

Fecha Aceptación: Agosto 2019 


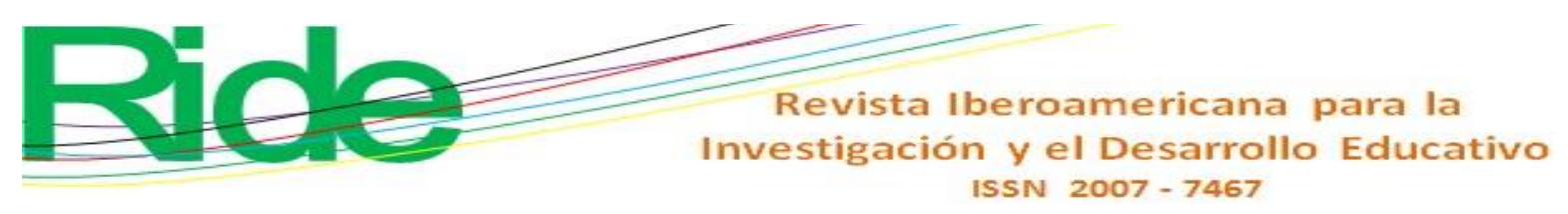

\section{Introducción}

A lo largo de la historia las distintas instituciones que conforman la sociedad deben ir cambiando constantemente para ajustarse a las necesidades propias de cada época. Un ejemplo de ello lo constituyen las universidades, entidades educativas sobre las cuales recae la responsabilidad tanto de generar el conocimiento científico más actualizado como de transmitirlo a las generaciones futuras. Así ha sido desde siempre, como sucedió en la academia de Platón o como acontece en instituciones más modernas, como la Universidad de Bolonia en Italia, la Universidad de París en Francia y la Universidad de Oxford en Inglaterra, las cuales se destacan por poseer una estructura formal y estudios reconocidos (Buchbinder, 2011; Gómez, 1998).

En efecto, en todo el recorrido histórico que va desde la Antigüedad hasta el presente, las universidades se han caracterizado por su flexibilidad para adaptarse a los diferentes escenarios que se han ido presentando. De hecho, en la actualidad la revolución tecnológica ha exigido a las universidades incluir dentro de su ámbito formativo el uso de recursos multimedia para mejorar la calidad educativa y para formar profesionistas con altos estándares en torno a las destrezas digitales. Al respecto, Navarro (2007) comenta: "Las tecnologías de la información y comunicación (TIC) han desarrollado un potencial extraordinario en las últimas décadas configurando un nuevo tipo de sociedad en la era de la información" (p. 6).

Las universidades, por ser sistemas educativos

abiertos, no pueden estar ajenas a los cambios que ocurren en su entorno

Perrenoud (2010) define una competencia como "una capacidad de actuar de manera eficaz en un tipo definido de situación; capacidad que se apoya en conocimientos, pero no se reduce a ellos" (p. 7). Las competencias involucran un conjunto de saberes (conocimientos, habilidades y actitudes) con relación a un desempeño particular, desde el enfoque de las TIC en estudiantes de Educación Superior implica las capacidades para utilizar estas herramientas tecnológicas de forma eficaz como parte de su proceso formativo (para aprender), y para su aplicación en el ámbito profesional.

En este contexto, y como lo comenta Cabero (2007), el uso masivo de Internet y el libre acceso a enormes cantidades de información desde cualquier dispositivo móvil ha servido para crear nuevos espacios de interacción: "Nos movemos en un nuevo espacio, el ciberespacio, en una nueva sociedad, la cibersociedad, en una nueva cultura, la cibercultura, con un nuevo dinero, el dinero electrónico, y en unas nuevas estancias educativas, los centros virtuales" (p. 5). Por ello, es 


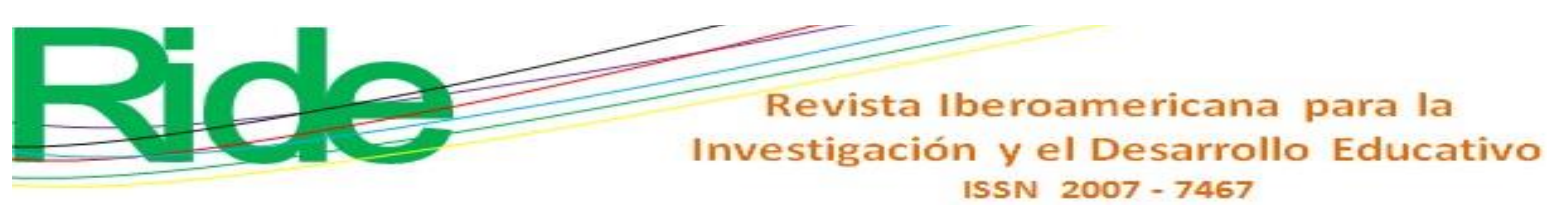

necesario que los nuevos profesionistas puedan aprovechar las ventajas que ofrecen las tecnologías más recientes para optimizar los procesos productivos y para resolver problemas propios de las diferentes áreas del saber (Amador, 2013).

Explicado lo anterior, se puede indicar que en el presente trabajo se ha procurado determinar cuáles son las competencias que en torno a las TIC manejan los estudiantes del Instituto Tecnológico José Mario Molina Pasquel y Henríquez (TECMM), campus Puerto Vallarta. El propósito es suministrar información relevante en lo institucional para el diseño de estrategias formativas en las áreas que requieran mayor consolidación en cuanto a la manera que usan los estudiantes las TIC. Con ello se espera ayudar a dar respuesta a los retos que impone el sector productivo a las instituciones de educación superior, especialmente en todo lo vinculado con la necesidad de formar profesionales que sean capaces de adaptarse y resolver problemas en sus respectivas especialidades.

\section{Objetivo general}

- Analizar el desarrollo de competencias en el uso de las TIC de alumnos del TECMM, campus Puerto Vallarta, con el fin de establecer niveles de apropiación según los estándares ISTE y diseñar estrategias que coadyuven a la mejora educativa.

\section{Objetivos específicos}

- Revisar el estado de la cuestión de propuestas de estándares en competencias para el uso de las TIC en estudiantes.

- Diagnosticar las competencias tecnológicas para el uso de las TIC de estudiantes del TECMM, campus Puerto Vallarta, con base en los estándares ISTE.

- Describir el desarrollo de competencias para el uso de las TIC de los alumnos del TECMM, campus Puerto Vallarta, para proponer recomendaciones y estrategias de mejora.

\section{Metodología}

La presente fue una investigación de tipo analítica y transversal, con un enfoque cuantitativo, ya que las variables se cuantificaron para ser analizadas. Asimismo, el estudio se basó en los principios de la investigación descriptiva porque se examinaron y describieron aspectos de la realidad que permitieron determinar las competencias en cuanto al uso de las TIC de alumnos 


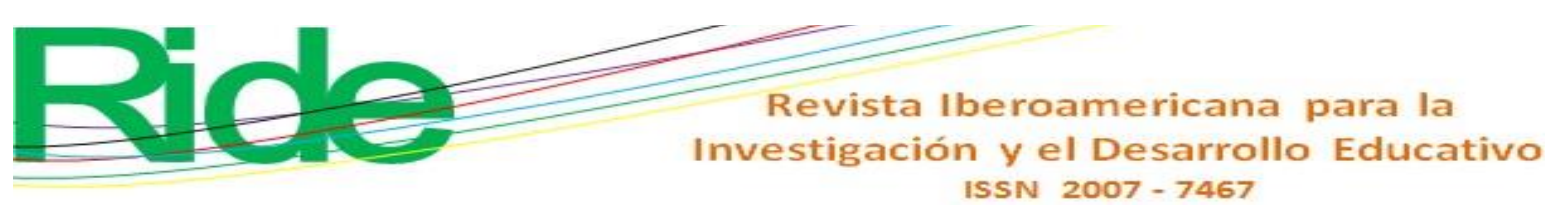

del TECMM, campus Puerto Vallarta. Igualmente, y con los resultados recabados, se propusieron estrategias para promover el empleo más eficiente de las TIC en los procesos de enseñanza y aprendizaje.

En cuanto al método, este fue deductivo porque se emplearon modelos preestablecidos que permitieron hacer un recorrido que inició en lo general y culminó en lo particular; pero, al mismo tiempo, fue inductivo porque para la recolección y el análisis de los datos se trabajó con una muestra probabilística que permitió generar conclusiones más generales. De forma general, a continuación, se presentan los pasos seguidos en esta investigación:

1. Revisión del estado de la cuestión sobre estándares para el empleo de las TIC en alumnos universitarios y sobre instrumentos para analizar el uso de las TIC.

2. Determinación del tipo de instrumento a administrar.

3. Selección de la muestra y administración del instrumento.

4. Procesamiento de datos, obtención de resultados y elaboración de conclusiones.

\section{Revisión del estado de la cuestión sobre estándares para el empleo de las TIC en alumnos universitarios y sobre instrumentos para analizar el uso de las TIC}

Una vez revisado el estado de la cuestión, se decidió adoptar como marco teóricoconceptual la propuesta de los estándares en competencias TIC para estudiantes de la International Society for Technology in Education (ISTE, 2007).

Figura 1. Estándares ISTE para estudiantes

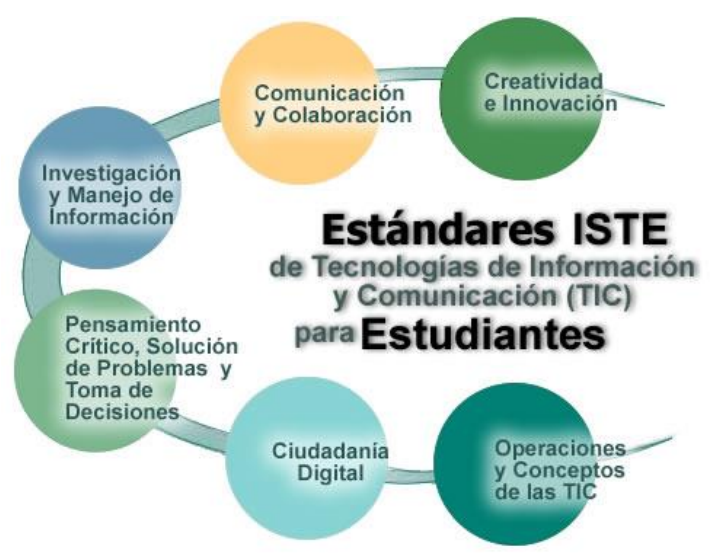

Fuente: International Society for Technology in Education (2007) 


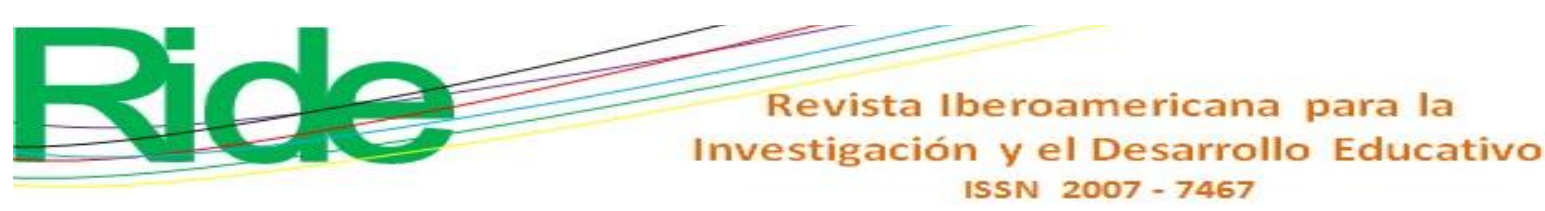

Esta propuesta comprende seis estándares: 1) creatividad e innovación, 2) comunicación y colaboración, 3) investigación y manejo de información, 4) pensamiento crítico, solución de problemas y toma de decisiones, 5) ciudadanía digital y 6) operaciones y conceptos de las TIC. Sobre estos estándares se especifican indicadores de competencias de uso de las TIC. De acuerdo con la International Society for Technology in Education (2007), estos indicadores son relevantes porque en ellos se especifica "lo que los estudiantes deberían saber y ser capaces de hacer para aprender efectivamente y vivir productivamente en un mundo cada vez más digital” (p. 2). A continuación, se describen estos indicadores.

a. Creatividad e innovación

Este indicador se refiere a cuando los estudiantes demuestran pensamiento creativo, construyen conocimiento y desarrollan productos y procesos innovadores utilizando las TIC. En otras palabras, los estudiantes cumplen con las siguientes labores:

- Aplican el conocimiento existente para generar nuevas ideas, productos o procesos.

- Crean trabajos originales como medios de expresión personal o grupal.

- Usan modelos y simulaciones para explorar sistemas y temas complejos.

- Identifican tendencias y prevén posibilidades.

b. Comunicación y colaboración

Este indicador se refiere a cuando los estudiantes utilizan medios y entornos digitales para comunicarse y trabajar de forma colaborativa, incluso a distancia, para apoyar el aprendizaje individual y contribuir al aprendizaje de otros. Los estudiantes cumplen con lo siguiente:

- Interactúan, colaboran y publican con sus compañeros, con expertos o con otras personas, empleando una variedad de entornos y de medios digitales.

- Comunican efectivamente información e ideas a múltiples audiencias, usando una variedad de medios y de formatos.

- Desarrollan una comprensión cultural y una conciencia global mediante la vinculación con estudiantes de otras culturas.

- Participan en equipos que desarrollan proyectos para producir trabajos originales o resolver problemas. 


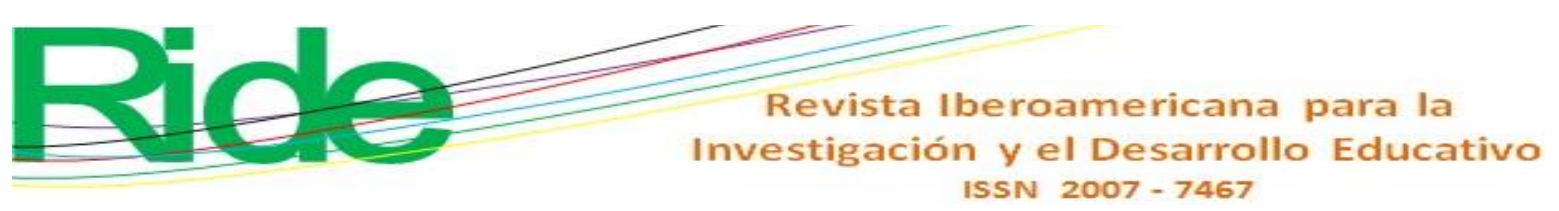

c. Investigación y manejo de información

Este criterio tiene que ver con estudiantes que aplican herramientas digitales para obtener, evaluar y usar información. Los estudiantes realizan estas tareas:

- Planifican estrategias que guían la investigación.

- Ubican, organizan, analizan, evalúan, sintetizan y usan éticamente información a partir de una variedad de fuentes y medios.

- Evalúan y seleccionan fuentes de información y herramientas digitales para realizar tareas específicas, basadas en su pertinencia.

- Procesan datos y comunican resultados.

d. Pensamiento crítico, solución de problemas y toma de decisiones

Esta variable se refiere a cuando los estudiantes usan habilidades de pensamiento crítico para planificar y conducir investigaciones, administrar proyectos, resolver problemas y tomar decisiones informadas usando herramientas y recursos digitales apropiados. Los estudiantes, por tanto, cuplen las siguientes asignaciones:

- Identifican y definen problemas auténticos y preguntas significativas para investigar.

- Planifican y administran las actividades necesarias para desarrollar una solución o completar un proyecto.

- Reúnen y analizan datos para identificar soluciones y/o tomar decisiones informadas.

- Usan múltiples procesos y diversas perspectivas para explorar soluciones alternativas.

e. Ciudadanía digital

En este criterio los estudiantes comprenden los asuntos humanos, culturales y sociales relacionados con las TIC y practican conductas legales y éticas. En síntesis, los estudiantes realizan este tipo de actividades :

- Promueven y practican el uso seguro, legal y responsable de la información y de las TIC.

- Exhiben una actitud positiva frente al uso de las TIC para apoyar la colaboración, el aprendizaje y la productividad.

- Demuestran responsabilidad personal para aprender a lo largo de la vida.

- Ejercen liderazgo para la ciudadanía digital. 


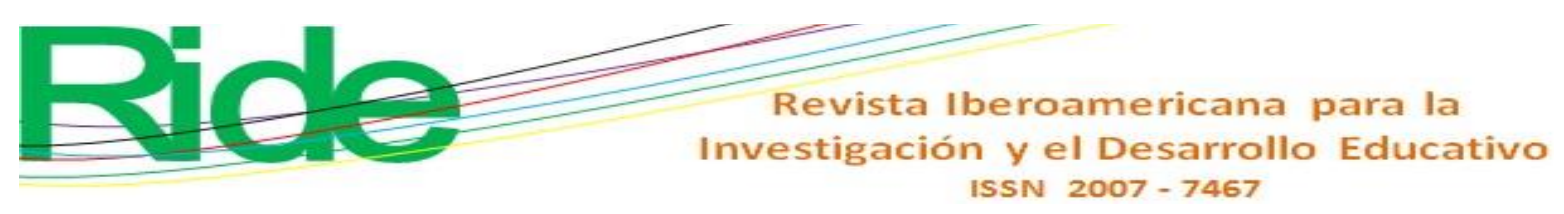

Tabla 2. Datos de la muestra por carrera (estratificada)

\begin{tabular}{|l|r|r|}
\hline Carrera & Población & Muestra \\
\hline Ingeniería en Gestión Empresarial & 516 & 100 \\
\hline Ingeniería en Sistemas & 201 & 39 \\
\hline $\begin{array}{l}\text { Informática y Tecnologías de la } \\
\text { Información }\end{array}$ & 50 & 10 \\
\hline Electromecánica & 232 & 45 \\
\hline Arquitectura & 289 & 56 \\
\hline Gastronomía & 317 & 60 \\
\hline Total & 1605 & 310 \\
\hline
\end{tabular}

Fuente: Elaboración propia

\section{Procesamiento de datos, obtención de resultados y elaboración de conclusiones}

Los datos obtenidos a partir del formulario se exportaron a una hoja de cálculo en donde se generaron gráficas que fueron analizadas y sirvieron para emitir conclusiones.

\section{Resultados}

A continuación, se presentan los resultados recabados en las encuestas aplicadas a los alumnos participantes:

Figura 2. Alumnos encuestados por sexo

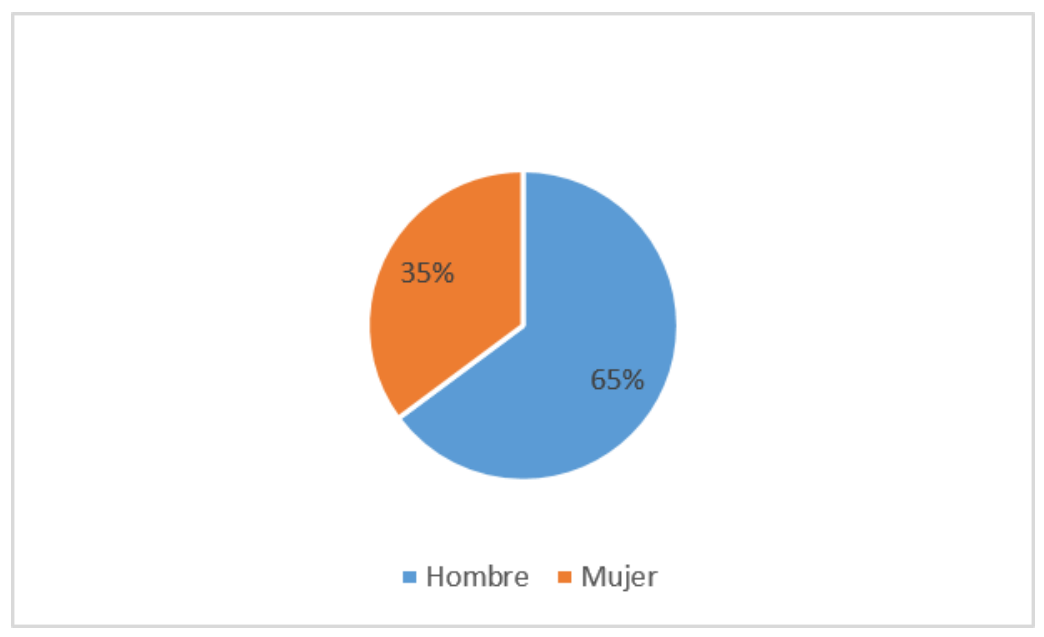

Fuente: Elaboración propia 


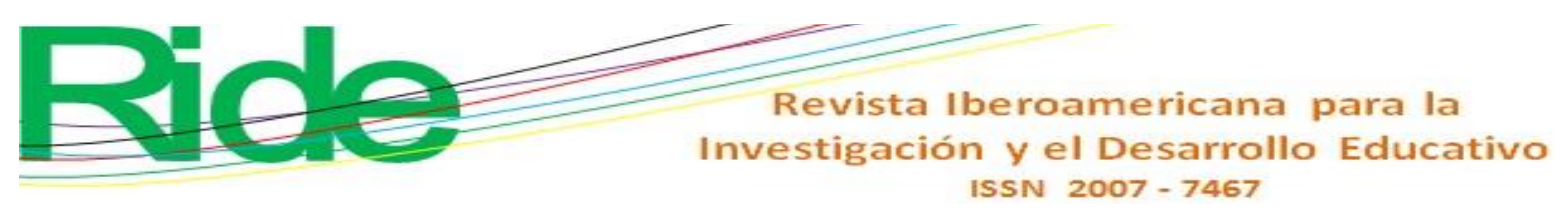

En la figura 2 se presentan los resultados obtenidos según las 310 encuestas realizadas. En dicha figura se aprecia que $65 \%$ (210 estudiantes) fueron hombres, mientras que el restante $35 \%$ (109) fueron mujeres.

Figura 3. Promedio de edades de alumnos encuestados

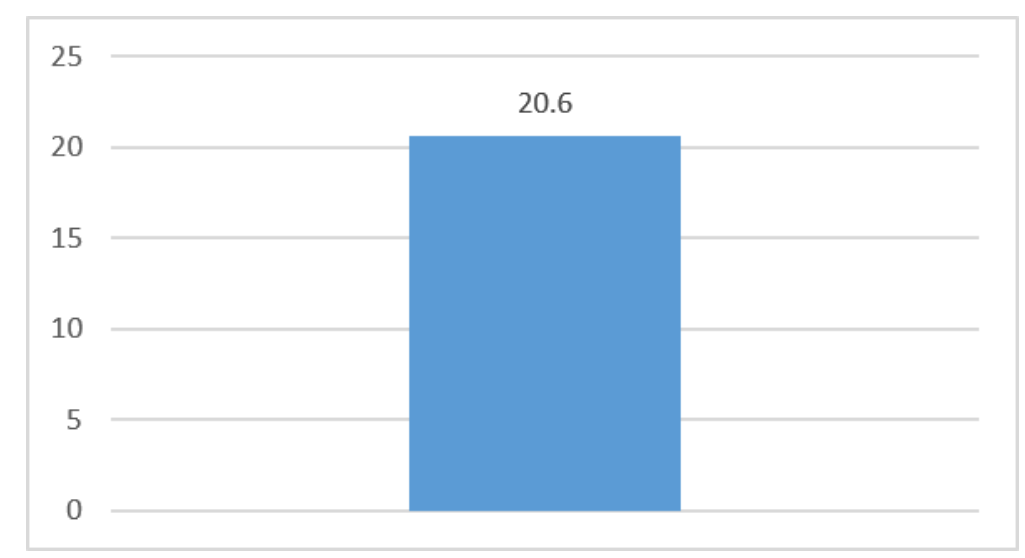

Fuente: Elaboración propia

El promedio de edades de los alumnos encuestados fue de 20.6 años; en cuanto a los rangos de edad, los de menor edad tenían 18 años, mientras que hubo una persona de 46 años (mayor rango de edad).

Figura 4. Equipo de cómputo

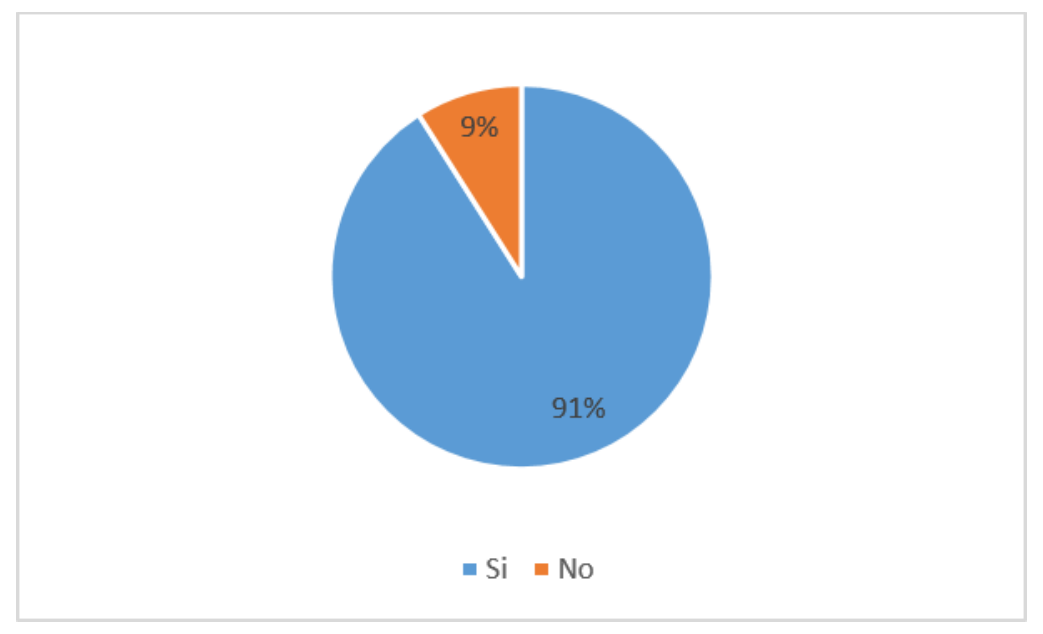

Fuente: Elaboración propia

De los 310 encuestados, 282 (91\%) indicaron que tenían equipo de cómputo propio, mientras que 28 alumnos ( $9 \%$ ) respondieron de forma negativa. Este dato es relevante, ya que es un indicador del acceso de los alumnos a las TIC y de la importancia que esto tiene para cursar 


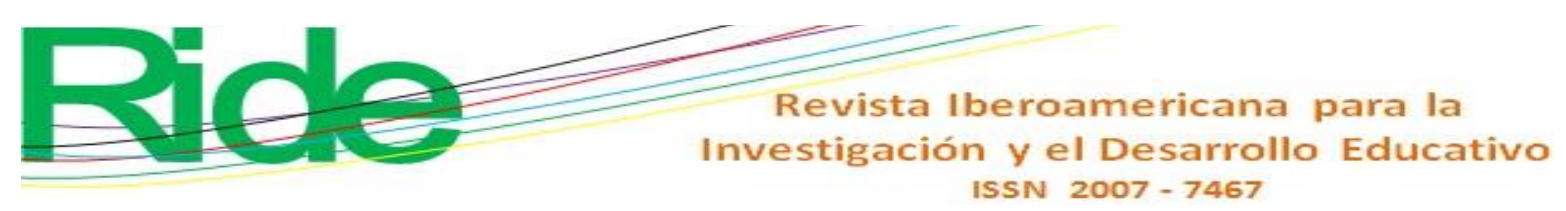

estudios superiores.

Figura 5. Computadora de escritorio en casa

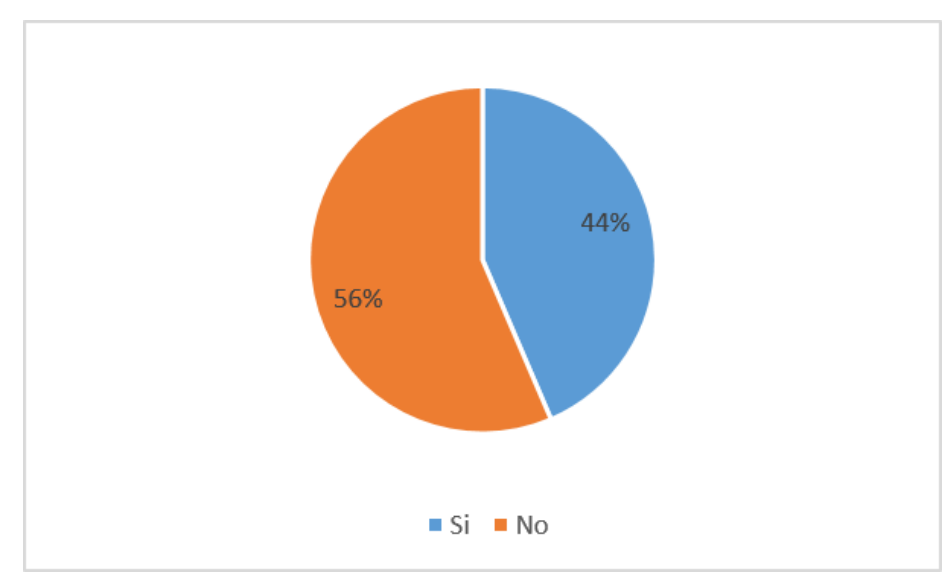

Fuente: Elaboración propia

En la figura 5 se presentan los resultados de los alumnos que tienen computadora de escritorio en casa. En tal sentido, 135 (44\%) señalaron que sí tenían este equipo, mientras que 175 $(56 \%)$ respondieron de forma negativa.

Figura 6. Computadora personal (laptop)

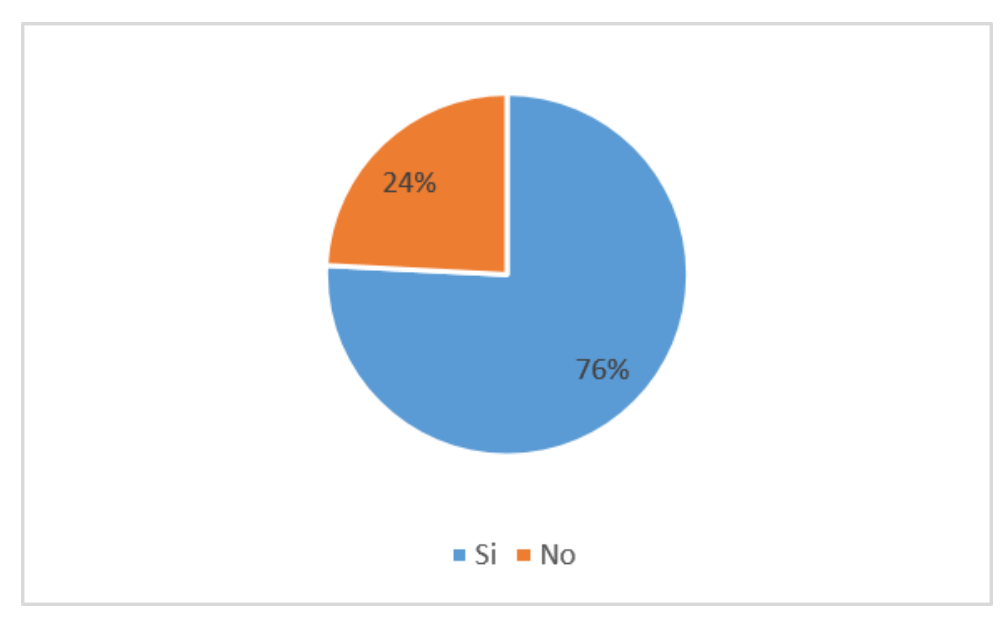

Fuente: Elaboración propia

En la figura 6 se presentan los resultados de los alumnos que tienen computadora personal o laptop. Al respecto, 235 (76\%) sí cuentan con ese dispositivo, mientras que 75 (24\%) no tienen una laptop. Si se compara este resultado con la información enseñada en la figura 5 , se puede 


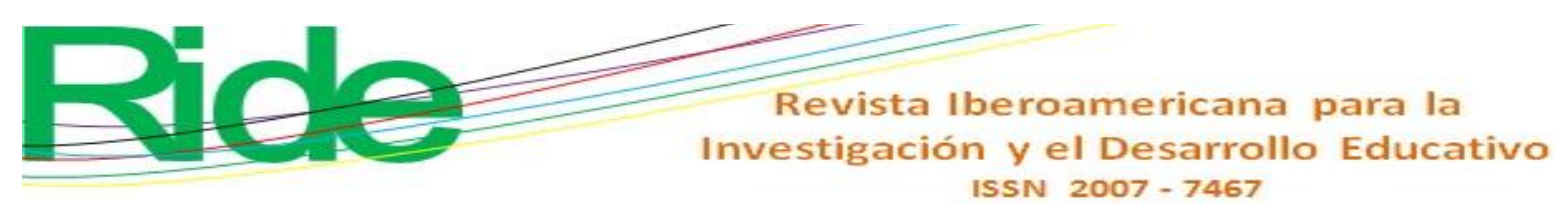

observar que son más los estudiantes que tienen una laptop en comparación con aquellos que tienen computadora de escritorio.

Figura 7. Servicio de internet en casa

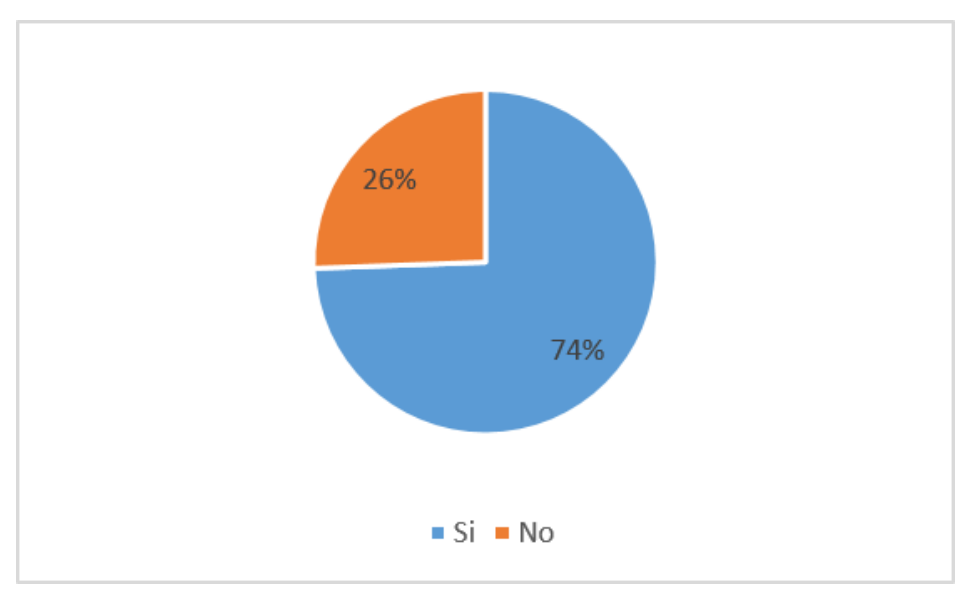

Fuente: Elaboración propia

En la figura 7 se evidencia que 230 alumnos (74 \%) tienen internet en su casa, mientras que 79 estudiantes $(26 \%)$ no cuentan con este servicio.

Figura 8. Alumnos encuestados por carrera

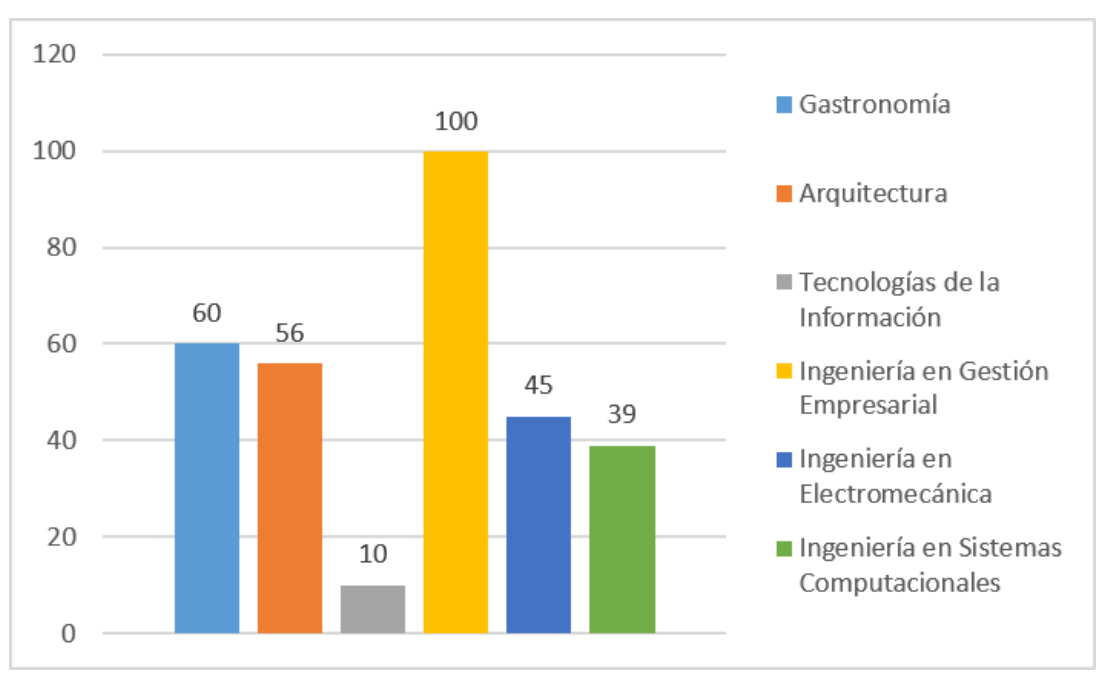

Fuente: Elaboración propia

En la figura 8 se presenta la distribución de la muestra total por carrera. Según los datos recabados, la mayor cantidad de alumnos encuestados (100) estudiaban la carrera Gestión 


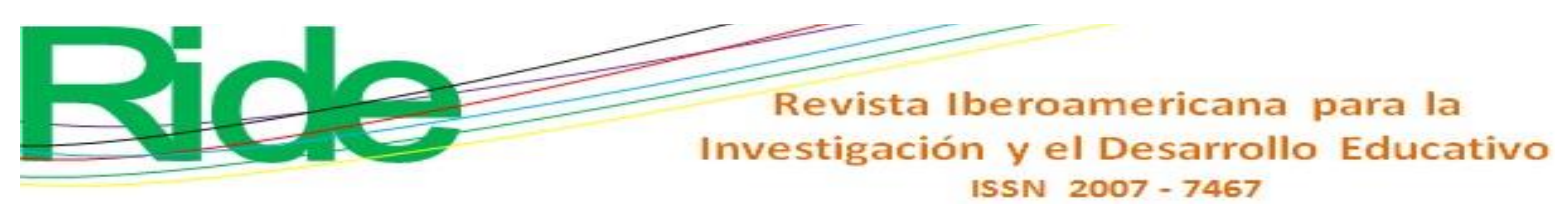

Empresarial, mientras que de Tecnologías de la Información solo participaron 10 estudiantes debido a que para la fecha esta era una carrera nueva.

Figura 9. Resultados generales por dimensión

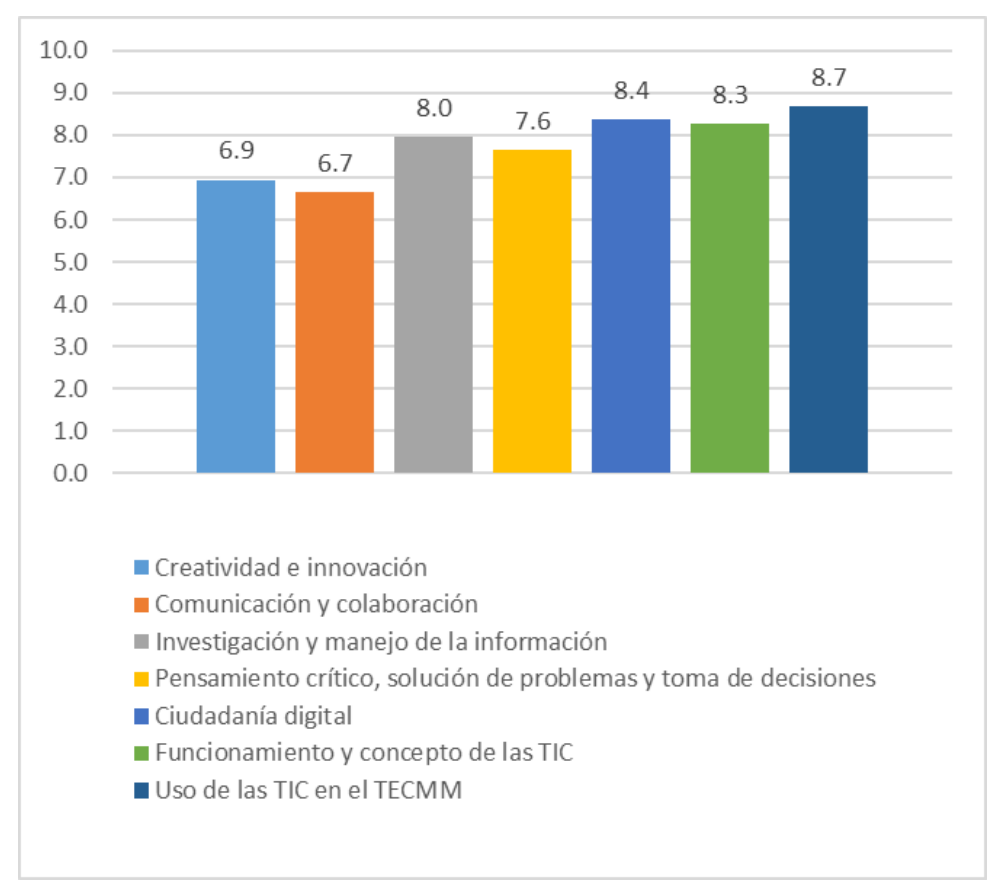

Fuente: Elaboración propia

La figura 9 muestra los resultados generales de todas las carreras por cada una de las dimensiones analizadas. Los valores más bajos se obtuvieron en la dimensión comunicación y colaboración con 6.7 de 10 posibles, seguido de creatividad e innovación con 6.9. Los valores más altos se hallaron en uso de las TIC en el TECMM. De forma general, se destaca que los alumnos usan de forma adecuada las herramientas institucionales relacionadas con las TIC, tales como la plataforma de educación a distancia y el sistema interno para trámites académicos. 


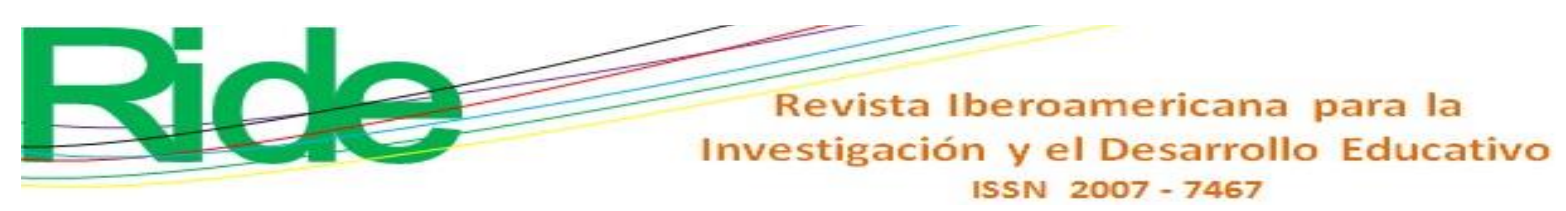

En la figura 11 se muestran los resultados promedio por carrera obtenidos en la dimensión creatividad e innovación. Los valores más bajos se consiguieron en ingeniería en Electromecánica con 6.5, y los más altos en Arquitectura con 7.7. Como ya se indicó en la figura 10, se debe tomar en cuenta que estos últimos utilizan herramientas especializadas en TIC para el diseño arquitectónico, de ahí que desarrollen habilidades relacionadas con la creatividad y la innovación.

Figura 12. Comunicación y colaboración por carrera

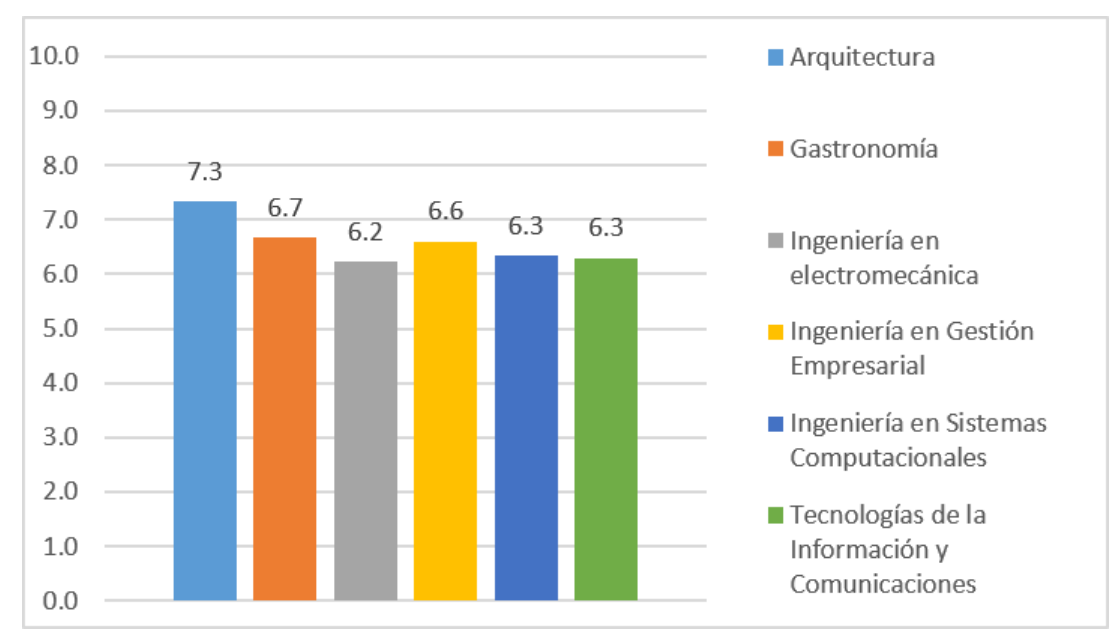

Fuente: Elaboración propia

En la figura 12 se ven los resultados promedio obtenidos por carrera en la dimensión comunicación y colaboración. Los valores más bajos se encontraron en ingeniería en Electromecánica con 6.2, y los más altos en Arquitectura con 7.3, resultados similares a la dimensión creatividad e innovación. 


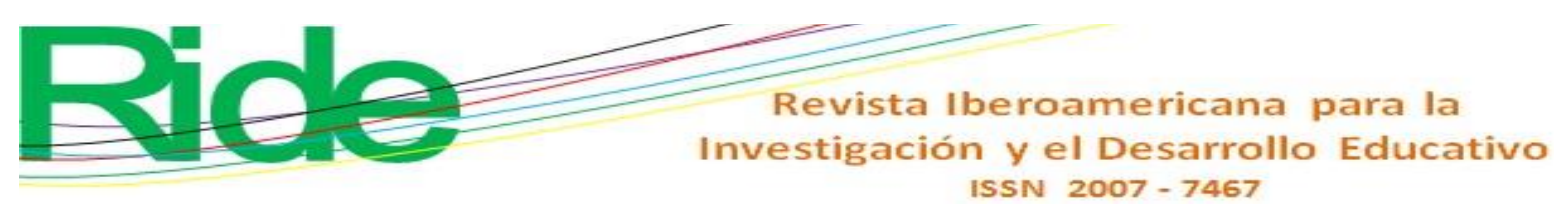

Figura 13. Investigación y manejo de la información por carrera

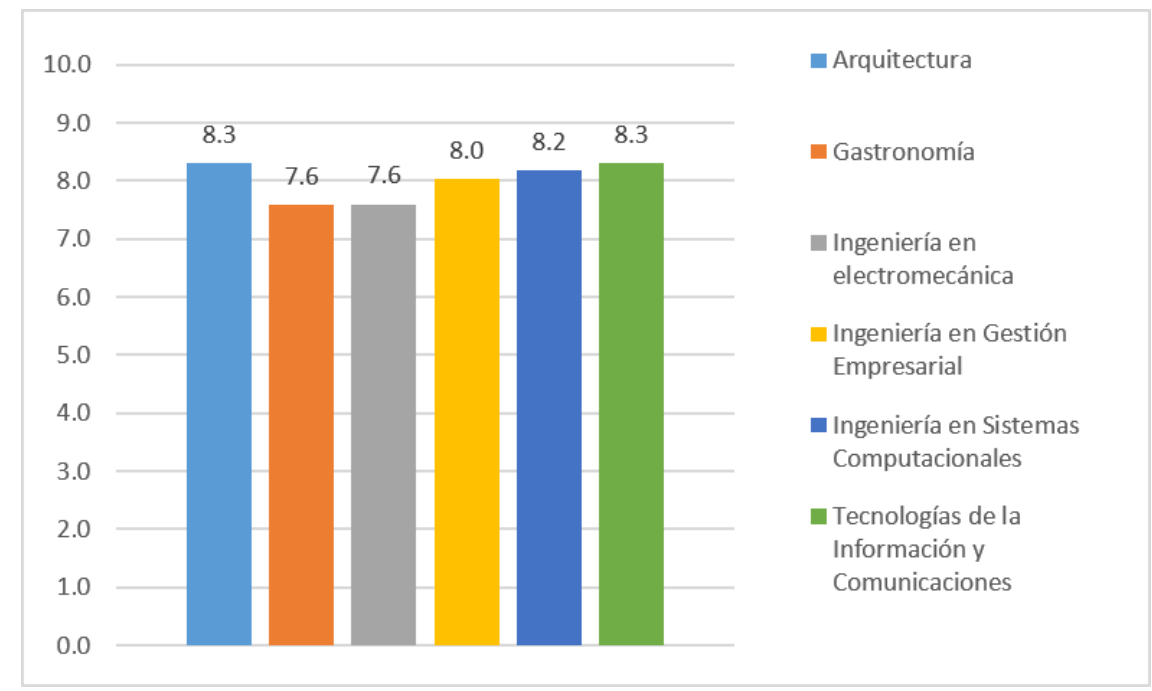

Fuente: Elaboración propia

En la figura 13 se observan los resultados de la dimensión investigación y manejo de la información. Los valores más bajos estuvieron en Gastronomía y en ingeniería en Electromecánica con 7.6, y los más altos en Arquitectura y en Tecnologías de la Información con 8.3.

Figura 14. Pensamiento crítico, solución de problemas y toma de decisiones por carrera

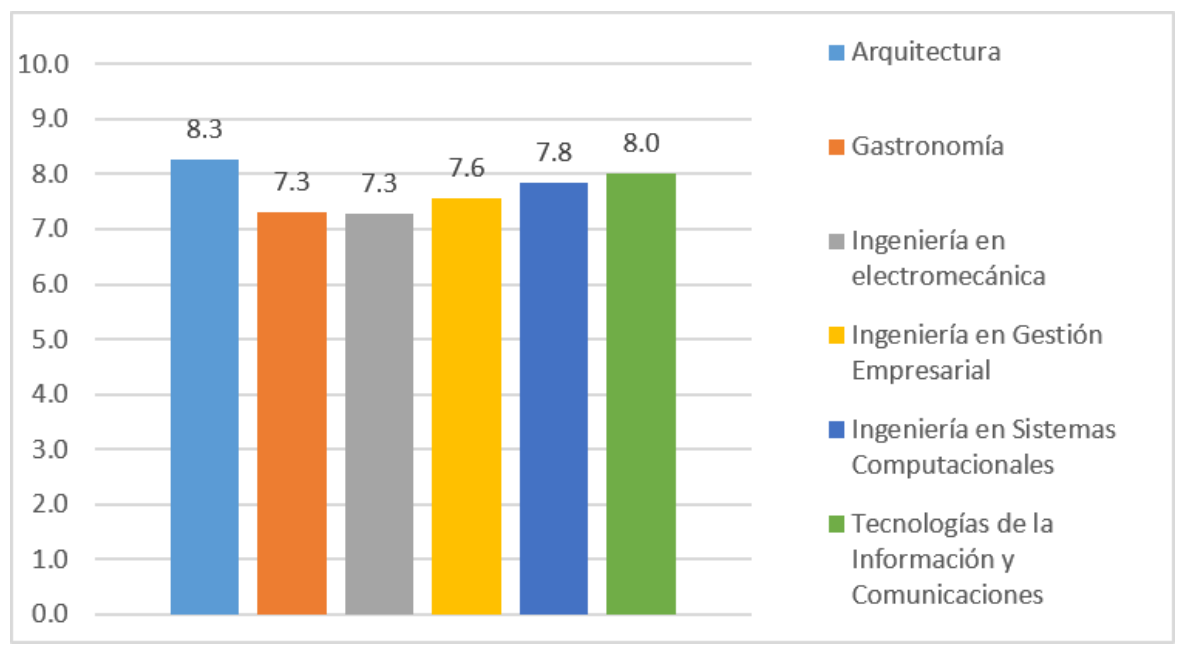

Fuente: Elaboración propia 


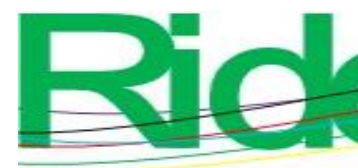

\section{Revista Iberoamericana para la Investigación y el Desarrollo Educativo \\ ISSN $2007-7467$}

La figura 14 enseña los resultados de la dimensión pensamiento crítico, solución de problemas y toma de decisiones. Los valores más bajos se obtuvieron en Gastronomía y en ingeniería en Electromecánica con 7.3, y los más altos en Arquitectura con 8.3.

Figura 15. Ciudadanía digital por carrera

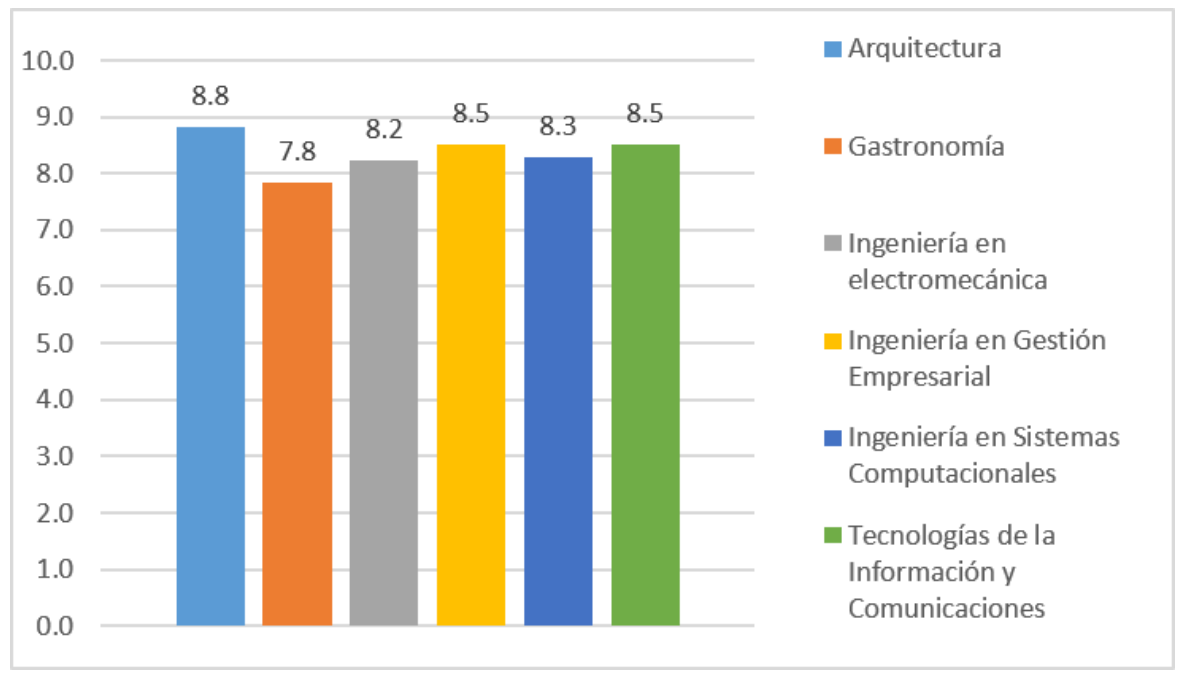

Fuente: Elaboración propia

En la figura 15 se muestran los resultados promedio por carrera en la dimensión ciudadanía digital. Los valores más bajos se obtuvieron en Gastronomía con 7.8, y los más altos en Arquitectura con 8.8.

Figura 16. Funcionamiento y conceptos de las TIC por carrera

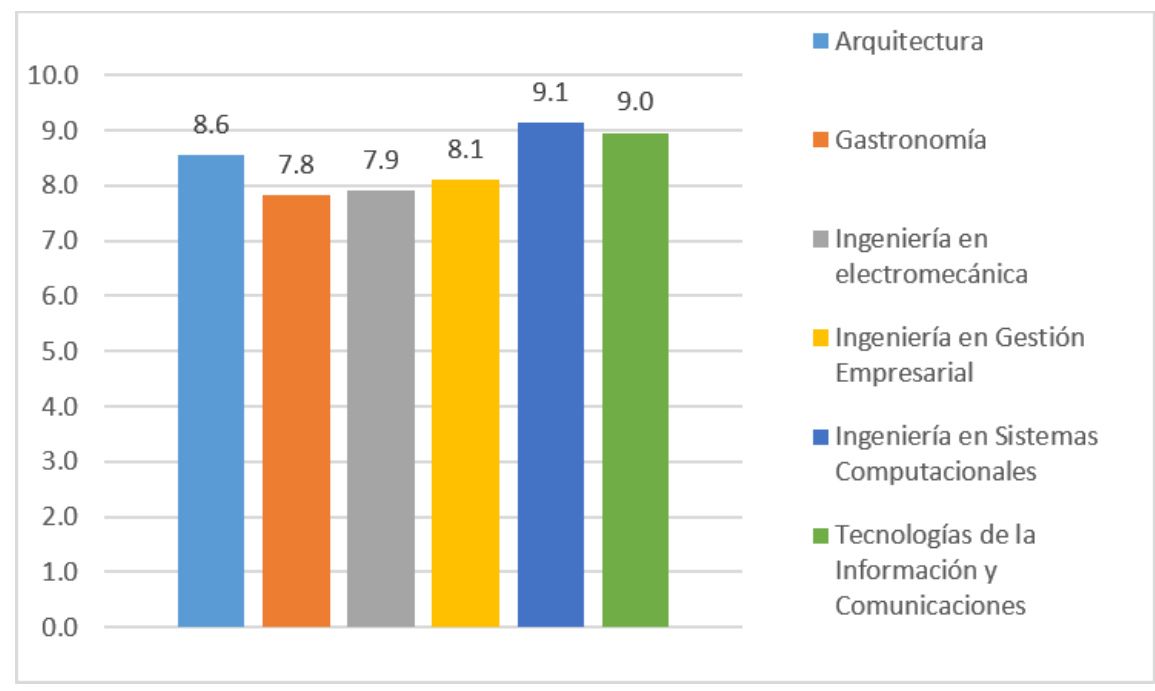

Fuente: Elaboración propia 


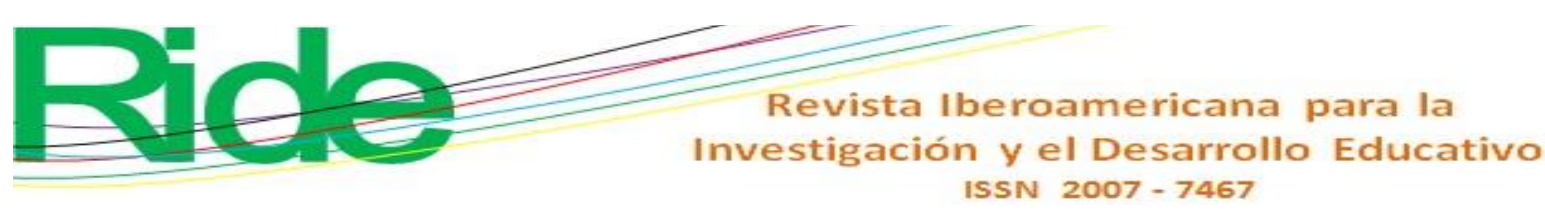

En la figura 16 se observan los resultados promedio obtenidos por carrera en la dimensión funcionamiento y concepto de las TIC. Los valores más bajos se consiguieron en Gastronomía con 7.8, y los más altos en ingeniería en Sistemas Computacionales con 9.1, seguido por Tecnologías de la Información y Comunicaciones con 9.0, lo cual resulta coherente con el perfil profesional de los estudiantes de estas carreras.

Figura 17. Uso de las TIC en el TECMM por carrera

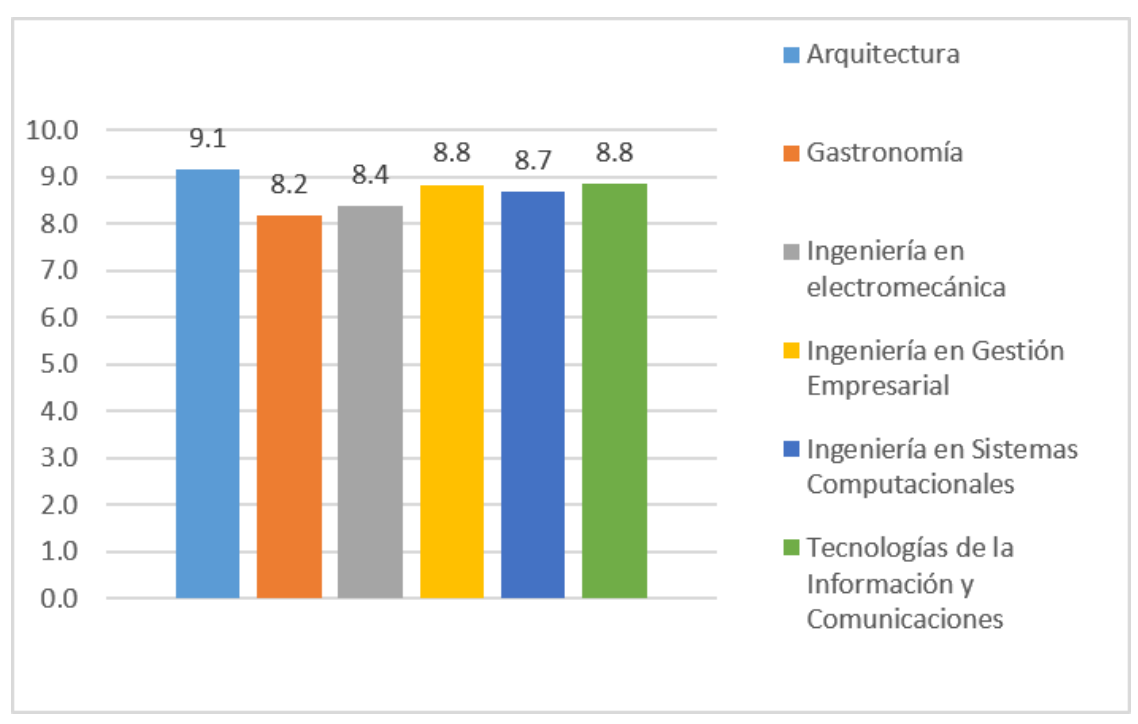

Fuente: Elaboración propia

En la figura 17 se aprecian los resultados promedio obtenidos por carrera en la dimensión uso de las TIC en el TECMM. Los valores más bajos estuvieron en Gastronomía con 8.2, y los más altos en Arquitectura con 9.1.

\section{Discusión}

En cuanto al análisis de competencias para el uso de las TIC de estudiantes universitarios, cabe destacar que de 310 alumnos encuestados únicamente 28 (9\%) no tienen computador personal, bien sea de escritorio o portátil. Asimismo, 79 (25\%) no cuentan con servicio de internet en casa. Estas cifras se relacionan con la infraestructura y el nivel de penetración que tienen las TIC en el ámbito de la educación superior.

Por otra parte, se puede indicar que los resultados promedio obtenidos en cada una de las dimensiones fue de 7.8 de 10 posibles, lo que demuestra un alto nivel de apropiación de los alumnos 


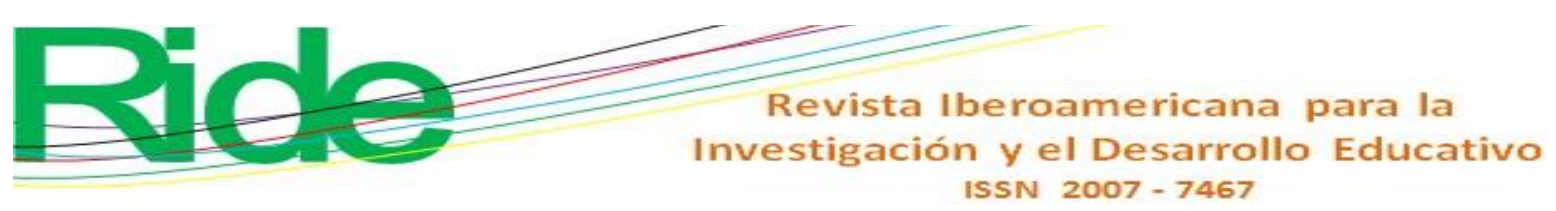

de las TIC en la institución. Si estas cifras se intepretan según lo considerado por la Unesco (2008), se puede afirmar que los alumnos del instituto se hallan en el nivel tres de integración, el cual hace referencia al acceso a computadora, internet y otros recursos TIC, así como a capacidades para la gestión de la información, uso académico de internet, comunicación a través de diferentes dispositivos tecnológicos y dominio de diferentes herramientas TIC como apoyo para la formación académica. Esta categorización tiene que ver con que la mayoría de los alumnos encuestados nacieron hacia finales de la década de 1990, es decir, durante la difusión masiva de la Web, de ahí que se les considere nativos digitales (Prensky, 2001).

De forma resumida, se puede indicar que los principales resultados de las dimensiones analizadas fueron los siguientes: 8.3 en funcionamiento y concepto de las TIC; 8 en investigación y manejo de información, y 6.7 en comunicación y colaboración. Si bien este último resultado es inferior a 7, de forma general se puede asegurar que cubre algunos aspectos del nivel cuatro propuesto por la Unesco (2008), el cual se refiere al acceso a computadora e internet en la casa y en la escuela, acceso a otros recursos TIC (como videoproyectores, cámaras, escáner, teléfonos inteligentes), uso de las herramientas TIC para la autogestión, habilidades para la gestión de la información y uso de plataformas de aprendizaje en línea. De hecho, según lo recabado en el presente estudio, se pudo constatar que todos los alumnos tienen acceso a una computadora de escritorio y a internet en el instituto (la mayoría incluso cuenta con estas herramientas en su casa). Además, en la institución los alumnos tienen acceso a videoproyectores, cámaras y escáner.

Sobre el uso de las herramientas TIC para la autogestión y las habilidades para la gestión de la información, en las dimensiones investigación y manejo de la información se obtuvieron resultados de 8.0, y en pensamiento crítico, solución de problemas y toma de decisiones, así como en ciudadanía digital se consiguieron resultados de 7.6 y 8.4 respectivamente. Asimismo, en la dimensión uso de las TIC en el TECMM (donde se preguntó por el uso de la plataforma de educación a distancia institucional) se consiguieron valores de 8.7. Esto quiere decir que también se cubre en gran medida el nivel 4 de acuerdo a los estándares TIC de la Unesco (2008).

En lo concerniente a los resultados por carrera, los valores promedio más bajos de todas las dimensiones se obtuvieron en Gastronomía y en Electromecánica con 7.4, y los valores más altos en Arquitectura con 8.3. Igualmente, las dimensiones más bajas, de forma general, fueron comunicación y colaboración con 6.7 y creatividad e innovación con 6.9 , mientras que el valor más alto fue uso de las TIC en el TECMM con 8.7.

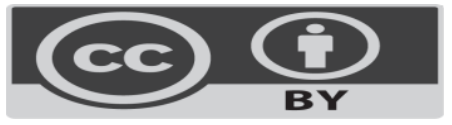




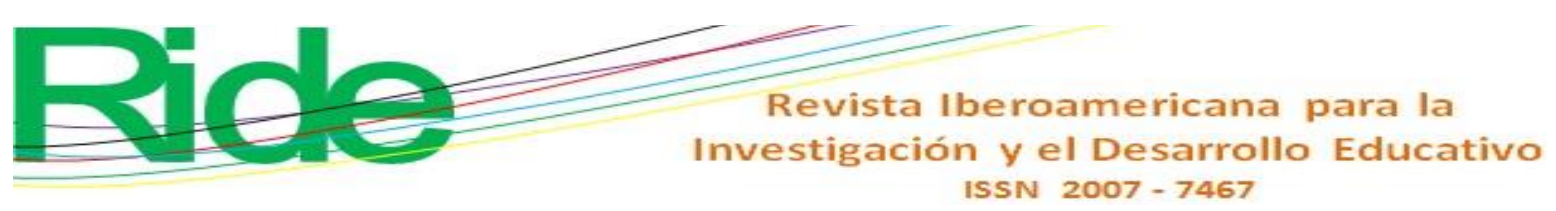

autogestión del aprendizaje mediante las TIC; 3) definir estrategias para el aprendizaje de software especializado según los perfiles profesionales de cada carrera, y 4) fomentar el uso de plataformas en línea para impulsar la autogestión, la planificación y la organización del tiempo, así como la responsabilidad individual frente al proceso de aprendizaje.

\section{Referencias}

Amador, C. (2013). Diagnóstico de competencias tecnológicas en la educación superior. EL caso del Insituto Tecnológico Superior de Puerto Vallarta. Revista Iberiamericana de $\begin{array}{llll}\text { Educación, } & 62(3), & \text { 1-14. } & \text { Recuperado de }\end{array}$ http://rieoei.org/deloslectores_bbdd.php?pageNum_deloslectores=0\&totalRows_deloslect ores $=121 \&$ id_tema $=55$.

Amador, C. (2015). Instrumento para diagnosticar competencias tecnológicas a estudiantes de educación superior. En Santillán Campos, F. (ed.), Experiencias educativas en instituciones de nivel superior en Latinoamérica (pp. 82-93). Guadalajara, Jalisco: CENID. Recuperado de https://dialnet.unirioja.es/descarga/libro/652197.pdf.

Buchbinder, P. (2011). La universidad: breve introducción a su evolución histórica. Recuperado de

http://www.instrumentalia.com.ar/uploads/archivos/evolucion_historica_educ_sup.2011.p df.

Cabero, J. (2007). Las necesidades de las TIC en el ámbito educativo: oportunidades, riesgos y necesidades. Tecnología y Comunicación Educativas, 21(45), 4-19.

Cabero, J., Llorente, M. y Marín, V. (2010). Hacia el diseño de un instrumento de diagnóstico de "competencias tecnológicas del profesorado" universitario. Revista Iberoamericana de Educación, 52(7),1-12.

Gómez, G. (1998). La universidad a través del tiempo. México: Universidad Iberoamericana.

Hernández, R., Fernández, C. y Baptista, P. (2010). Metodología de la investigación (5. ${ }^{a}$ ed.). México: Mc Graw Hill.

International Society for Technology in Education (2007). ISTE nets. Retrieved from https://id.iste.org/docs/pdfs/nets_2007_spanish.pdf?sfvrsn=2

Llorente, M. y Cabero, J. (2010). Desarrollo de un instrumento sobre competencias TIC en alumnos universitarios. En Congreso Euro-Iberoamericano de Alfabetización Mediática y Culturas Digitales Sevilla: Universidad de Sevilla (pp. 1-13). Universidad de Sevilla. 


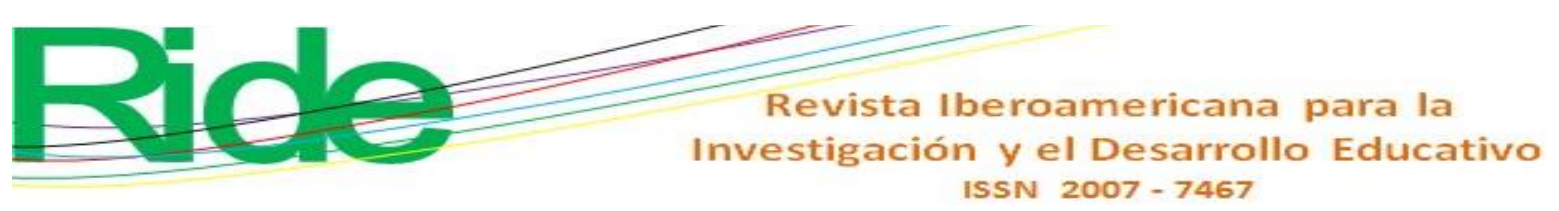

López, M. (2007). Uso de las TIC en la educación superior en México. Un estudio de caso. Apertura: Revista de Innovación Educativa, 7(7), 19.

López, R. (2008). Acceso, uso y apropiación de las tecnologías de la información y comunicación (TIC) en los estudiantes universitarios de la UNAM. X Congreso Nacional de Investigación Educativa (pp. 1-11). Veracruz, México.

Navarro, D. (2007). La era del "homo tecnologicus". Escritura Pública, (43), 6-13. Recuperado de http://www.notariado.org/liferay/c/document_library/get_file?folderId=12092\&name=DL FE-10629.pdf.

Oviedo, C. y Campo-Arias, A. (2005). Aproximación al uso del coeficiente alfa de Cronbach. Revista Colombiana de Psiquiatría, 34(4), 572-580.

Perrenoud, P. (2010). Diez nuevas competencias para enseñar. México: Graó.

Prensky, M. (2001). Digital Natives Digital Immigrants. MCB University Press, 1-6.

Rangel, A. y Peñalosa, E. (2013). Alfabetización digital en docentes de Educación superior: construcción y prueba empírica de un instrumento de evaluación. Pixel-Bit Revista de Medios y Dducación, (43), 9-23.

Unesco (2008). Estándares de competencia en TIC para docentes. Lóndres: UNESCO. 


\begin{tabular}{|l|l|}
\hline Rol de Contribución & Autor (es) \\
\hline Conceptualización & Carlos Miguel Amador Ortíz \\
\hline Metodología & Carlos Miguel Amador Ortíz \\
\hline Software & Leticia Velarde Peña \\
\hline Validación & Leticia Velarde Peña \\
\hline Análisis Formal & Carlos Miguel Amador Ortíz \\
\hline Investigación & Carlos Miguel Amador Ortíz (principal) \\
\hline Recursos & Noticia Velarde Peña (apoyo) \\
\hline Curación de datos & Leticia Velarde Peña \\
\hline Escritura - Preparación del & Carlos Miguel Amador Ortíz \\
\hline Escritura - Revisión y edición & Carlos Miguel Amador Ortíz (principal) \\
\hline Visualización & Leticia Velarde Peña \\
\hline Supervisión & Leticia Velarde Peña \\
\hline Administración de Proyectos & Carlos Miguel Amador Ortíz \\
\hline Adquisición de fondos & Carlos Miguel Amador Ortíz \\
\hline
\end{tabular}

